Research Article

\title{
Integration of Museum User Behavior Information Based on Wireless Network
}

\author{
Shuangji Liu $\mathbb{D D}^{1,2}$ Yongzhong Yang, ${ }^{1}$ and Yiwei Wang ${ }^{2,3}$ \\ ${ }^{1}$ Sichuan University, Chengdu 610065, China \\ ${ }^{2}$ Henan Finance University, Zhengzhou 451464, China \\ ${ }^{3}$ Segi University, Kuala Lumpur 47810, Malaysia \\ Correspondence should be addressed to Shuangji Liu; 2017325020032@stu.scu.edu.cn
}

Received 1 June 2021; Revised 1 July 2021; Accepted 2 July 2021; Published 10 July 2021

Academic Editor: Fazlullah Khan

Copyright ( $\odot 2021$ Shuangji Liu et al. This is an open access article distributed under the Creative Commons Attribution License, which permits unrestricted use, distribution, and reproduction in any medium, provided the original work is properly cited.

Online museum information resource systems are getting popular these days which allow the users to get detailed information about the objects of their interest, and the user preferences are stored to search for related artifacts considering his/her online behavior. The behavior of users browsing online is integrated to capture relevant information which is integrated into museum information resources. Unfortunately, present implementations have errors in integration and optimization system, so a wireless network-based museum user behavior information integration system is proposed to calculate the user's interest in museum's cultural relics. The user behavior information resource model is developed based upon the degree of user interest, and forgetting functions with different decay rates are employed to describe changes in the interest level. This information is then used to construct users' interest matrices. This matrix also contains information regarding the cultural relics that users have not yet visited. The system will introduce the interest weights of feature words to take the top features of the user behavior information for the integration of the users' behavior and to combine the feature vectors that can represent the overall trajectory. Moreover, those feature vectors are described that can represent the local trajectory into feature vector to identify the slow-moving sparse targets, which is then utilized for the integration of users' behavior information. The simulation tests prove that the proposed method can achieve low error in the integration process of user behavior information resources, thereby yielding good results.

\section{Introduction}

The rapid development of information technology in modern society has influenced every field of life including museum visitors. More and more people are taking interest in visiting online museums where they can find relics with interesting information. The study of user behavior information systems refers to various levels including different categories of museums based on user demand for information resources or interest. This is made possible through the use of computing devices, communication technology, digital multimedia, and other related high-tech means [1]. Wireless network technology has entered thousands of households [2], and it has enriched the overall amount of information in society. However, museum information resources currently available online are chaotic and scattered.
In order to obtain high-value museum information resources in a relatively short period of time, information from multiple users could be captured online and integrated into museum information resource space [3]. Integrating museum information resources based on user behavior not only fulfills users' actual needs for museum information resources but would also improve information resources to become in line with users' generic needs, thereby making up for the lack of information resources in the museum's existing collections, and would also improve the low-level duplication of museum information resources.

Zhou [4] proposed the construction of a digital resource integration model for libraries, archives, and museums based on linked data. Through understanding the basic principles of linked data, the study explained the feasibility, process, general framework, and specific integration of LAM 
(Library, Archives, and Museum) digital resources based on linked data. The LAM cloud service platform considers the basic framework and analyzes specific cases based upon linked data. On this basis, the author proposed that when implementing the LAM digital resource, it is necessary to pay attention to the library user interaction mode, open licensing standards, maintenance of linked data, and conversion of existing data. In another study, Chen [5] designed the interactive user behavior information interface for the functional entrance of the Meishan Nuo mask exhibits based on the Hunan Provincial Museum APP and analyzed and discussed the interactive design requirements and multiple scheme choices for the said purpose. The Meishan mask represents the most important carriers of the Meishan Nuo culture which is one of the ancestral Chinese cultures and is a significant part of the Huxiang history in central south China. In the actual process of the interactive design related to the functional entrance of the Meishan Nuo mask exhibits, the ideas and methods for specifically decomposing key factors according to business needs and user needs are explained which includes the process of negotiation with product managers, market operations, and development engineers. This results in the completion of the first level of the drafts of pages, the secondary pages, and interactive visual drafts of the system. The aforesaid method has the dilemma of the large number of errors and long timeconsuming optimization of the museum user behavior information resource integration. In order to solve the above problems, a wireless network-based museum user behavior information integration is proposed in this paper, and the validity conclusion is reached through research.

\section{Integration of Museum User Behavior Information Resources}

The museum user behavior information resource cluster is divided into several clusters. Initially, the user trajectory is calculated and the association rules between the two are obtained by extracting the minimum support and trust information of the user [6]. These association rules are utilized to calculate the user's preference value for a particular museum information resource and the cosine similarity measurement method is used to calculate the similarity between users' interests, so as to realize the integration of user behavior information.

Assuming that $\bar{A}$ means the time period $l$ containing $n$ object collections and $m$ individual user dynamic files, then $C_{q}$ will represent a subsequence where the user behavior information resource cluster is divided into $q$ clusters as given by

$$
C=\left\{C_{1}, C_{2}, \cdots, C_{q}\right\}
$$

Now, assuming that $L_{i}$ represents the behavior trajectory of the user $i$ [7], then the number of the cluster to which user $i$ belongs at the time period $l$ can be calculated by

$$
L_{i}=\left(C_{i, T-l+1}, \cdots, C_{i, T-l}, C_{i, T}\right), i=1,2, \cdots, m,
$$

where $C_{i, T-k} \in C, k=0,1,2, \cdots, l-1, l \geq 2$.
Using association rule technology, the minimum support [8] and minimum trust information of the users is extracted to derive the association rules between the two. According to the association rules, if the behavior trajectory of users is $r_{j, T-l+1}, r_{j, T}, \cdots, r_{j, T-l}$, then it would mean that the behavior cluster of the user at that particular time is $r_{j, T}$ [9].

Assume that $P_{x}$ represents a collection of museum information resources with significant preferences of a user $x$ and $P_{i}$ represents a collection of museum information resources with significant preferences of user $i$, and the union of museum information resource collections with significant preferences of user $x$ and user $i$ denoted by $U_{x, i}$ could be calculated as given in

$$
U_{x, i}=P_{x} \cup P_{i}
$$

Similarly, assuming that $N_{x}$ represents a collection of museum information resources where user $x$ has no preference value in the union $U_{x, i}$, then the calculation formula is

$$
N_{x}=U_{x, i}-P_{x}
$$

For any particular museum's information resource $P_{j} \in U_{x, i}$, the preference of user $x$ for information resource $P_{j} \in N_{x}[10]$ could be evaluated as given in

$$
\theta_{x, j}=\left\{\begin{array}{l}
\omega_{x}\left(P_{j}\right) \\
\theta_{x}
\end{array},\right.
$$

where the first case is when the customer $x$ preference value for information resources is significant while the second case represents the vice versa. On the basis of the above calculations, the cosine similarity measurement method is used to calculate the similarity between user $x$ and user $i$ to formulate the integration of museum user behavior information resources as given by

$$
\Phi_{x, j}^{\prime}=\frac{\sum_{n \in M_{j}} \operatorname{sim}(x, i) \times \theta_{x}}{\sum_{n \in M_{j}}(|\operatorname{sim}(x, i)|)},
$$

where $M_{j}$ represents the collection of information similar to the museum's information resource $P_{j}, \operatorname{sim}(x, i)$ indicates the similarity of behavior between user $x$ and user $i$, and $\theta_{x}$ indicates user $x$ preference value for museum information resources $j$.

The structure diagram of user behavior information resource integration is shown in Figure 1.

\section{Integration and Optimization of Museum User Behavior Information Resources}

The process of creating, integrating, and optimizing user behavior information resources constitutes three steps which are elaborated in the following subsections.

\subsection{Construction of the Museum User Behavior Information} Resource Interest Model. The user's interest in the keywords of a certain type of cultural relics in a museum is estimated by considering the time that the user takes in reading the 


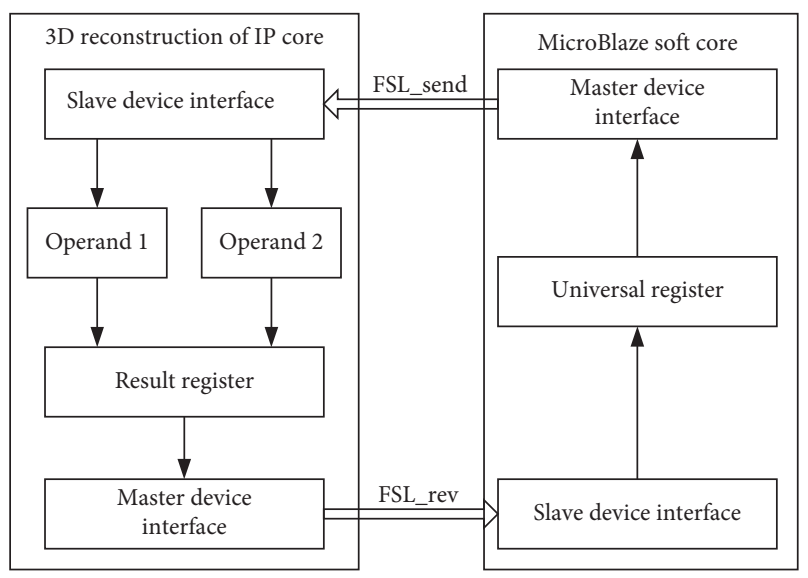

FIGURE 1: User behavior information resource integration structure diagram.

introductory document while visiting the museum's online website. The system uses forgetting functions with different decay rates to describe changes in user's interest [11] while constructing the user behavior information resource interest model.

In real life, while visiting an online museum, if a user reads the introduction of a certain cultural relic collection, this suggests that the user is interested in the information resource, and the length of time reading is directly proportional to the user's interest in that particular resource. The degree of user's interest takes into account the length of the relic's profile and the user's reading speed. Mathematically, a user $x$ 's interest denoted by $I_{\text {interests }}$ in museum's cultural relic $c$ could be calculated by equation (7) as follows:

$$
I_{\text {interests }}=\frac{1}{R_{\text {rate }}},
$$

where $R_{\text {rate }}$ represents the relative speed of the user $x$ browsing the museum's cultural relics $c$ profile which is calculated as follows:

$$
R_{\text {rate }_{x, c}}=\frac{\left(L_{\text {length }} / T_{\text {totaltime }}\right)}{\bar{r}_{x}} .
$$

In the formula, $L_{\text {length }}$ and $T_{\text {totaltime }}$ represent the length of the profile of relic $c$ and the time taken by the user $x$ to read it, respectively, while $\bar{r}_{x}$ represents the average speed of the user $x$ which is estimated as per

$$
\bar{r}_{x}=\frac{\sum_{\text {rate }_{x, c}}\left(L_{\text {length }} / T_{\text {totaltime }}\right)}{\left|d_{x}\right|} .
$$

Assume that $k_{e a}$ represents the probability that the web page $a$ belongs to the classification of cultural relics $e, I_{\text {interests }}$ shows the interest of the user $x$ in $a$, and $\psi_{\text {ba }}$ represents key feature words $b$ in profile page $a$; then the importance of the user's interest [12] in the keywords of the museum's cultural relics collection information resources is given by

$$
H_{\mathrm{bex}}=\sum_{a \in d_{x}} k_{\mathrm{ea}} \times \text { interests }_{s} \times \lambda_{\mathrm{ba}} .
$$

According to the user's interest in the keywords of a certain type of cultural relics in the museum, the user's interest in a certain type of similar cultural relics in the museum is represented by $u_{\mathrm{ex}}$ as given in

$$
u_{\mathrm{ex}}=\left(H_{1 \mathrm{ex}}, H_{2 \mathrm{ex}}, \cdots, H_{\text {mex }}\right), 1<a<m .
$$

Based on the above analysis, forgetting functions with different decay rates are used to describe changes in user's interests, and a museum user behavior information resource interest model is constructed [13]. The modeling formula is as follows:

$$
W_{f_{\mathrm{ex}}}=\frac{f_{\mathrm{ex}}}{F_{x}}+\left(1-u_{\mathrm{ex}}\right) \times \frac{1}{t_{\mathrm{cur}}-t_{x}+1}
$$

where $f_{\text {ex }}$ represents the user's $x$ visit frequency for $e$ relic collections, $F_{x}$ represents the user's $x$ visit frequency for all available relics collection, $t_{\text {cur }}$ represents the current display time of the system page, and $t_{x}$ represents the user's $x$ behavioral model construction or update time.

\subsection{Integration and Optimization of Museum User Behavior} Information Resources. Based on the construction of the user interest model for museum information resources, a user interest matrix is constructed which divides the users of online museum information resources into core users and noncore users, respectively, using K-means clustering algorithm and cosine similarity. These clusters are then utilized to calculate the interest weight for the feature words of a particular relic that the user has not yet visited. The top few features as per interest weight of the museum information resource are taken as pairs for the prediction of overall user behavior which is then utilized for the integration and optimization of museum user behavior information resources.

Assuming that $N$ represents the number of information resources available online which are visited by user collection $V$ and $M$ represents the total number of interest feature words of all users $K$. Based upon the abovementioned establishment, a user interest matrix of $M \times N$ is constructed. The users of online information resources are divided into two broad categories, i.e., core users and noncore users. The calculation formula for the core user set $V_{\text {core }}$ is given in

$$
V_{\text {core }}=\left\{x \mid x \in V, \frac{N_{\text {num }}}{M} w f_{\text {ex }}>\beta\right\} .
$$

where $N_{\text {num }}$ represents the number of nonempty user interest matrices constructed and $\beta$ represents the judgment threshold set in advance [14].

The process of clustering is carried out in two steps.

3.2.1. Clustering of Core Users. The K-means clustering algorithm is used to cluster the core users for information resources, and users with similar interests are divided into the same cluster. It may be noted that it is a very simple and easy to implement algorithm, yet it guarantees convergence and can easily adapt to new examples [15]. It also has the 
capability to generalize clusters of different sizes and shapes. The calculation formula for clustering in the proposed study is given in

$$
C=\left\{C_{1}, C_{2}, \cdots, C_{h}\right\},
$$

where $h$ represents the number of individual clusters in the cluster set.

3.2.2. Clustering of Noncore Users. The cosine similarity measurement method is used to cluster the information resources which are visited by noncore users. The calculation formula for this purpose is given in

$$
\operatorname{Sim}\left(x, c_{j}\right)=\frac{\vec{x} \times \overrightarrow{c_{j}}}{\|\vec{x}\| \times\left\|\overrightarrow{c_{j}}\right\|} .
$$

In order to prevent the cold start problem and to recommend diversified information resources to the users, the interest weights for the feature words of the museum's relic collection that the user has not yet visited are calculated which are then utilized to select the top features among them for the users' behavior prediction. It is then used to integrate and optimize museum user behavior information resources. The calculation formula is as follows:

$$
w_{i j}=\frac{\sum_{g \in M_{p}} \operatorname{Sim}(x, i) \times\left(\lambda_{\mathrm{ba}}-\bar{w}_{j}\right)}{\sum_{g \in M_{p}} \operatorname{Sim}\left(x, c_{j}\right)}+\bar{w}_{i},
$$

where $M_{p}$ represents the user with the highest interest feature similarity to user $x ; \bar{w}_{j}$ and $\bar{w}_{i}$ represent the average interest weight of the user against the top "h" users with the highest interest feature similarity, respectively.

\subsection{Abnormal Behavior Information Integration Containing} Slowly Moving Sparse Target User. In the process of integrating abnormal behavior information of slow-moving sparse target users, trajectory feature extraction is very critical. The feature vector contains the overall trajectory as well as the local trajectory, so the trajectory information of the slow-moving and sparse target user is described through the multidimensional feature vector. For this purpose, the trajectory is divided into trajectory segments of the same time period according to time and trajectories where the dividing points are described as follows:

$$
\begin{aligned}
Y^{i} & =\left\{\left(a_{j}^{i}, b_{j}^{i}\right) ; \gamma=1,2, \cdots, L_{i}\right\}, \\
\bar{Y}^{i} & =\left\{\left(a_{0}^{i}, b_{0}^{i}\right),\left(a_{L_{1}}^{i}, b_{L_{1}}^{i}\right), \cdots,\left(a_{L_{c}}^{i}, b_{L_{c}}^{i}\right)\right\},
\end{aligned}
$$

where $Y^{i}$ represents the trajectory while $i$ indicates the number of the tracks, $\left(a_{j}^{i}, b_{j}^{i}\right)$ represent the filter position of the $i$ th target, $L_{i}$ indicates the length of the $i$ th track, i.e., the number of track points, $c$ represents the number of segments dividing the trajectory according to the same time-division length, and finally $\bar{Y}^{i}$ represents the set of all division points.

Let $f_{z}(\cdot)$ be a feature extraction function, described as $z=1,2, \cdots, Z$, where $Z$ represents the total number of feature spaces; then $f_{z}$ maps each trajectory $Y^{i}$ to a $Q_{z}$-dimensional feature space, where each feature space is processed separately to prevent all feature spaces from being damaged in the normalization processing.

The feature extraction process starts from the actual scene and situation. Each feature space can roughly reflect the internal situation of the sample data. Combining several feature vectors can more accurately reflect the trajectory characteristics. The first feature is the direction and length of the trajectory of the slowly moving sparse target in the user behavior information. The current feature space vector is given as follows:

$$
p^{i}=\left[a_{L_{j}}^{i}-a_{0}^{i}, b_{L_{j}}^{i}-b_{0}^{i}, \tan \left(\frac{\left(b_{L_{j}}^{i}-b_{0}^{i}\right)}{\left(a_{L_{j}}^{i}-a_{0}^{i}\right)}\right)\right] .
$$

The second feature is the average position of the first trajectory. This feature is mainly used to distinguish the trajectory in different areas of the museum. The current feature space can then be described as

$$
z^{i}=\frac{1}{L_{i}} \sum_{j=0}^{L_{i}}\left(a_{j}^{i}, b_{j}^{i}\right)
$$

The third feature space is the initial position, the time length of the trajectory segment, and the segment direction. The trajectory is divided into $c$ segments evenly according to time and the current trajectory feature vector, as presented in

$$
\begin{aligned}
\bar{Y}^{i} & =\left\{\left(a_{0}^{i}, b_{0}^{i}\right),\left(a_{L_{1}}^{i}, b_{L_{1}}^{i}\right), \cdots,\left(a_{L_{c}}^{i}, b_{L_{c}}^{i}\right)\right\}, \\
\psi^{i} & =\left[\left(a_{0}^{i}, b_{0}^{i}\right), t^{i}, \omega^{i}, \theta_{1}^{i}, \cdots, \theta_{c-1}^{i}\right] .
\end{aligned}
$$

In the formula, $\left(a_{0}^{i}, b_{0}^{i}\right)$ represent the starting position; $t^{i}$ represents the time period; $\omega^{i}$ represents the direction of the first segment trajectory while $\theta_{j}^{i}, j=1,2, \cdots, c-1$ represents the deflection angle of other segment sequences. The above expressions not only approximate the shape of the trajectory but also reflect the direction of the trajectory.

The fourth feature space is the average speed, which is a supplement to the above three features. Combining the speed information with the above three feature spaces can effectively determine the shape of the trajectory. The average velocity feature space can be described as follows:

$$
\begin{aligned}
S^{i} & =\left[s_{1}^{i}, s_{2}^{i}, \cdots, s_{c}^{i}\right], \\
\vec{s}_{j}^{i} & =\left(\alpha_{j}^{i}, \beta_{j}^{i}\right), \\
s_{j}^{i} & =\operatorname{sqrt}\left(\alpha_{j}^{i} \times \alpha_{j}^{i}+\beta_{j}^{i} \times \beta_{j}^{i}\right) .
\end{aligned}
$$

After obtaining the trajectory characteristics of the slowmoving sparse target in the user behavior information, the abnormal trajectory integration of the target is realized through the $K$-clustering method, and the process is as follows: 
(1) Complete the clustering of the trajectory dataset and obtain cluster data

(2) Determine the cluster centers of different clusters

(3) The $K$-clustering method is used to gather clusters with cluster centers that are close together, to integrate similar clusters in order to accommodate abnormal trajectories of slow-moving sparse targets users' information

\section{Analysis of Simulation Results}

The simulation experiment data is collected from the home page of an online museum by using a collector, and the simulation experiment tool SDABAS DM is selected. According to the museum user behavior information resources, the effectiveness of the current study based on the wireless network is tested. The specific experimental configuration parameters utilized in the current study are shown in Table 1.

To evaluate the current study, three indicators of user behavior information integration and optimization systems are selected i.e., the average absolute error, the standard deviation of the average absolute error, and the average time consumption. The effectiveness of the current study is compared with two similar existing studies carried out by Zhou [4] and Chen [5].

In the simulation process, $70 \%$ of the collected dataset is selected for training purposes, and the remaining $30 \%$ is used for testing. Each group of experiments is performed 100 times, and the average values are taken as the final experimental result record. The comparison results of the average absolute error of different methods are summarized in Figure 2.

As depicted in Figure 2, the comparison result of the current study versus Zhou [4] and Chen [5] proves that, as the number of the nearest neighbors increases, the average absolute value of the proposed method shows a gradual decline, indicating that the accuracy of the integration and optimization of user behavior information resources is gradually improving while the average absolute error obtained by the proposed method is significantly lower than the other two comparison methods for all the values particularly when the number of the nearest neighbors increases to 20. The average absolute error of the three methods stabilizes when the number of the nearest neighbors grows beyond 60 . This shows that the current study performs significantly better and the integration accuracy increases with the increase of the nearest neighbors.

The effectiveness of the current research method lies in the integration process which mainly includes two stages. The first stage is the integration of user behavior information targets, and in the second stage, user behavior information targets are tracked while integrating the abnormal trajectories of user behavior. Simulation experiments are carried out to verify the reliability and practicability of the method presented in this paper.

The target shown in Figure 3 is integrated by utilizing the current study. The target appears in frames \#212 \#236 of the
TABLE 1: Simulation experiment configuration parameter table.

\begin{tabular}{lc}
\hline Configuration item & Parameter \\
\hline CPU & Intel core i5-4200M, 2.62 GHz \\
RAM & $4 \mathrm{~GB}$ \\
Operating system & Windows7 \\
Programming tools & Eclipse juno service release 1 \\
Programming language & $\mathrm{C}++$ \\
\hline
\end{tabular}

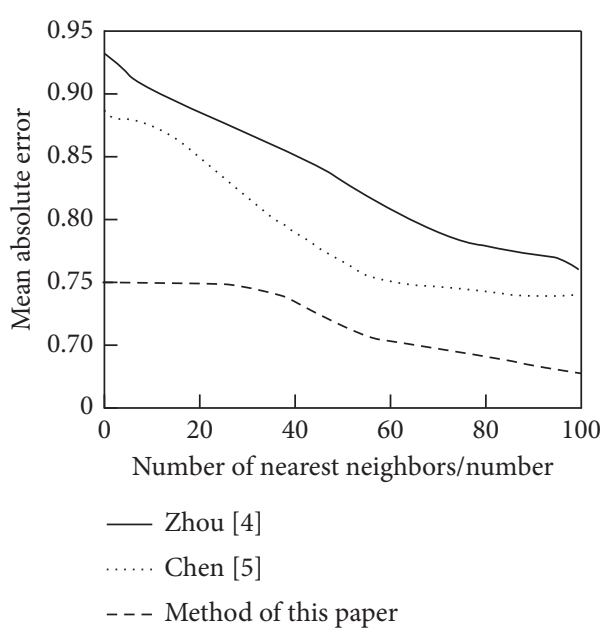

FIGURE 2: Comparison results of average absolute error indicators of different methods.

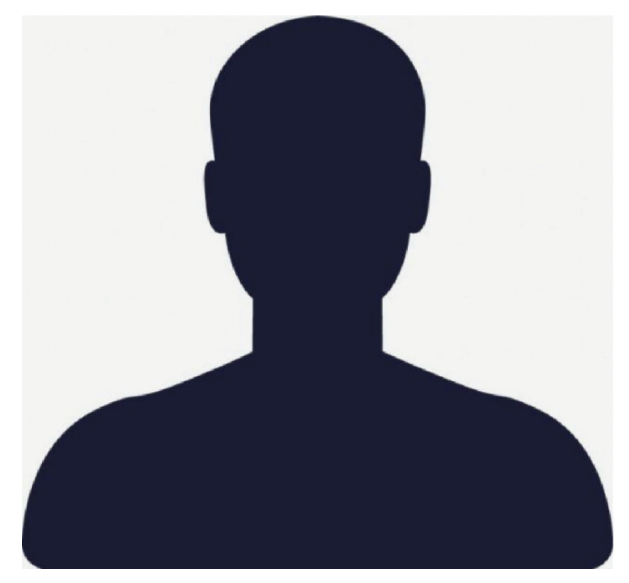

FIgURE 3: Tracking target.

video. During the monitoring process, the face of the tracking target is blocked. The 180 candidate regions obtained in frame \#212 are used to approximate the target template of the initial frame of the video sequence. Figure 4 describes the sparse coefficient vector obtained for the \#212 frame.

By analyzing Figure 4, it can be seen that most of the sparsity coefficient values are small, and only a small part of the sparsity coefficient values is high, which authorizes the effect of sparse approximation. At the same time, the advantage of using the sparse coefficient description is that the current frame is the closest to the target template, and the sparse coefficient error is 0.4015 , which is lower than the 


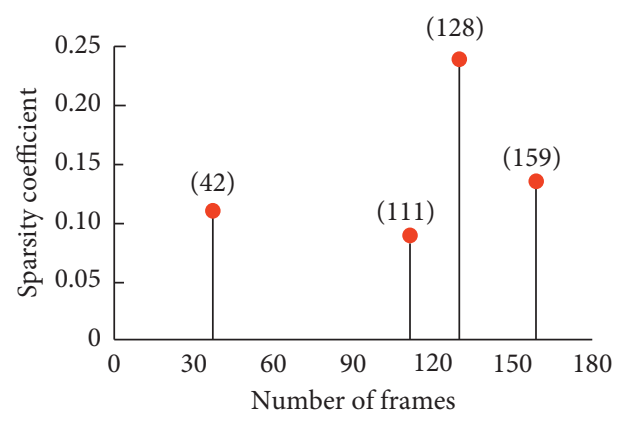

FIGURE 4: Frame 212's sparse representation result of the target template.

allowable error of 0.5 , indicating that the integration error of this method is acceptably low.

The entrance and exit of the museum are the only way for personnel to enter and exit the museum. The abnormal trajectory of museum user entrance information and user exit information is integrated. Under normal circumstances, users entering the museum have the same direction of operation at the entrance and exit, and they are not allowed to enter from the exit or leave from the entrance. Some tourists wander at the entrance and exit or walk too fast, etc. which are abnormal behaviors. Figures 5 and 6 describe the abnormal behavior at the entrance and exit of the museum.

Finally, the current study is compared against Zhou [4] and Chen [5], and the simulation results are described in Table 2 .

Results in Table 2 show that the current method only integrates one wrong entry, while Zhou [4] and Chen [5] have a high error integration rate for the abnormal user entrance and exit information which approve that the proposed method is reliable and practical.

For the sake of generality, Zhou [4] is compared with Chen [5] as well. Three methods are used to integrate the abnormal behavioral information of museum users on a certain day. The contour coefficient and the overall similarity are used as a measure of the reliability of the integration result.

Equation (26) gives the formula for the calculation of contour coefficient for a sample point $j$ :

$$
\varsigma_{s i l}(j)=\frac{n_{j}-m_{j}}{\max \left(m_{j}, n_{j}\right)},
$$

where $m_{j}$ is used to describe the average distance between the sample point $j$ and the sample points in the same cluster; $j$ is used to describe the minimum average distance between the sample point $\varsigma_{\text {sil }}(j) \in[-1,1]$ and the sample points in the other clusters. For $\varsigma_{\text {sil }}(j)=1$, it means that there is a big difference between the sample point $j$ and other sample points in this cluster and so $j$ is divided into the wrong cluster, so in general, it has a better integration effect of abnormal trajectories.

The overall similarity refers to the degree of aggregation within the sample data in each cluster, which can be obtained by the following equations:

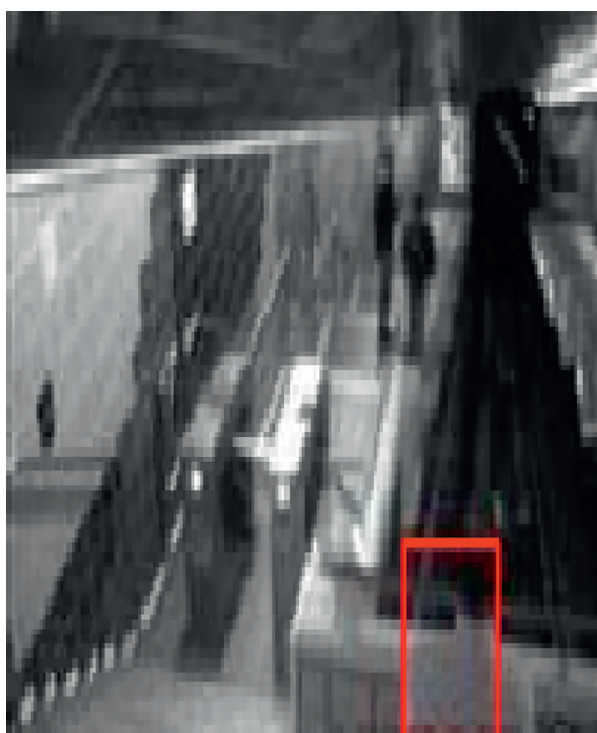

FIgURE 5: Abnormal behavior of users at the entrance of the museum.

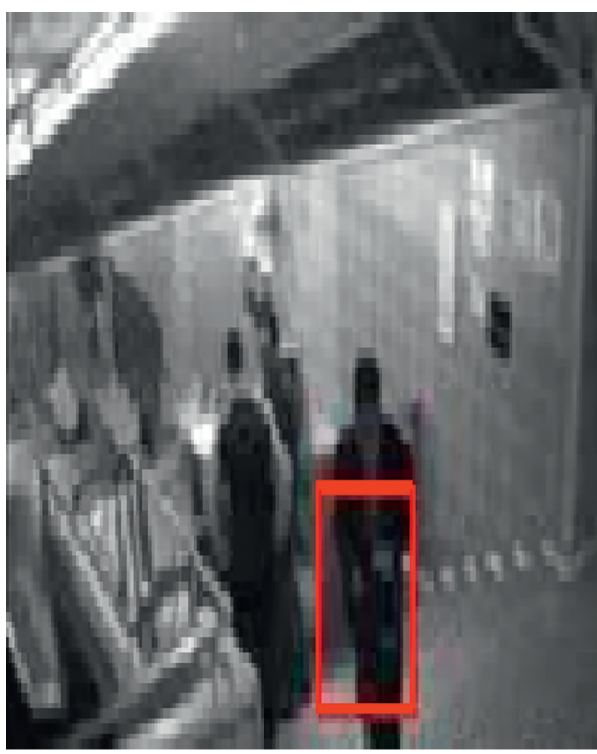

FIgURE 6: Abnormal behavior of users at the museum exit

$$
\begin{aligned}
\gamma_{\text {sim }}(j) & =\frac{\sum_{x \in j} d\left(x, C_{j}\right)}{X_{j}}, \\
\gamma_{\text {sim }}^{*} & =\sum_{j=1}^{N_{K}} \frac{x_{j}}{x} \gamma_{\text {sim }}(j),
\end{aligned}
$$

where $N_{K}$ describes the number of clusters. Equation (27) describes the average distance between all sample points in the cluster $j$ and the cluster center $C_{j}$, and equation (28) describes the weighted sum of the similarity of each cluster, as per the sample dataset. Generally, the smaller the value of the overall similarity, the better the integration effect of abnormal trajectories. 
TABLE 2: Integration results of abnormal trajectories of the three methods.

\begin{tabular}{lccccc}
\hline Method & $\begin{array}{c}\text { Total number of } \\
\text { tourists/person }\end{array}$ & $\begin{array}{c}\text { Entrance abnormal } \\
\text { track/person }\end{array}$ & $\begin{array}{c}\text { Wrong } \\
\text { integration/person }\end{array}$ & $\begin{array}{c}\text { Total number of } \\
\text { tourists left/person }\end{array}$ & $\begin{array}{c}\text { Exit abnormal } \\
\text { track/person } \\
\text { integration/ } \\
\text { person }\end{array}$ \\
\hline $\begin{array}{l}\text { Actual } \\
\text { results }\end{array}$ & 82 & 7 & 0 & 25 & 5 \\
$\begin{array}{l}\text { Current } \\
\text { study }\end{array}$ & 82 & 6 & 1 & 25 & 5 \\
$\begin{array}{l}\text { Zhou [4] } \\
\text { Chen [5] }\end{array}$ & 82 & 4 & 3 & 25 & 0 \\
\hline
\end{tabular}

TABLE 3: Comparison of the integration results of the three methods.

\begin{tabular}{lccc}
\hline Index & Current study & Zhou [4] & Chen [5] \\
\hline Average contour coefficient & 0.95 & 0.83 & 0.81 \\
Average similarity & 0.12 & 0.39 & 0.46 \\
\hline
\end{tabular}

Table 3 describes the integrated comparison results of the abnormal trajectories of the three methods.

The analysis of Table 3 shows that the average contour coefficient between the abnormal trajectory integration results of the current study and other trajectories is the highest, and the average similarity is the lowest, which is significantly better than Zhou [4] and Chen [5], indicating that the abnormal trajectory integrated by the proposed method is much reliable as compared to other trajectories.

\section{Conclusion and Prospect}

5.1. Conclusion. The integration and optimization of user behavior information regarding museum resources play an important role in providing interesting knowledgeable services to users and promoting the in-depth development and utilization of museum information resources [16, 17]. Considering the large errors and long-time consumption problems in the existing methods of integrating museum user behavior information, a wireless network-based method for integrating and optimizing museum user behavior information resources is proposed. Simulation experiments prove the effectiveness and superiority of the proposed method by achieving the following milestones:

(1) The museum user behavior information integration method based on the wireless network achieves the effect of sparse approximation where the integration error is significantly low

(2) The low integration rate error ensures the reliability and practicability of the proposed model

(3) The difference between the abnormal trajectory integrated by the current method and other trajectories is the largest which further endorses its reliability

\subsection{Prospect.}

(1) The user behavior information-based system can enhance cooperation between museums and can enrich the research in this field. Due to user privacy protection, researchers cannot obtain the original log files of user behavior information recorded in the museum. In the future, cooperation between museums and research institutions can provide a good platform for comprehensive research on museum user behavior information integration models to promote the progress of the research on the needs of museum users.

(2) The study may be expanded considering the physical museum visitor's experience along with the portal user. The users who physically visit a museum may give direct feedback regarding his/her interest which may be integrated with similar artifacts present online. Future researchers should pay attention to the relationship between physical museum users and archive portal users' behavioral rules and needs. In this way, both systems, i.e., the online museums and physical museums, will augment each other by providing a better user experience.

(3) Although the current study considers collecting the user interest information without user intervention, taking the data directly from the user can enhance the reliability and acceptability of the application. In this way, the user can actually figure out his/her interests which could be better utilized in further searching and recommendations for the same user as well as for others having similar interests.

(4) The interest of the user regarding an artifact is estimated considering the time he spends upon reading its details online. To augment the system, gadgets like a webcam can be utilized to ensure that the user is actually looking at the screen while reading about some artifact and is not busy with some other task. This will eliminate incorrect data accumulation by the application regarding the interest of the user while he is busy with some other task.

(5) The current study relies on K-means for the clustering job considering its simple implementation, scalability, and guaranteed convergence qualities. However, it is proposed that the current method may be replicated by employing other modern clustering algorithms like BIRCH, Affinity Propagation, and OPTICS.

\section{Data Availability}

The data used to support the findings of this study are available from the corresponding author upon request. 


\section{Conflicts of Interest}

The authors declare that they have no conflicts of interest.

\section{References}

[1] Y. Y. Chen, "Development of archives information resources and value display --a case study of guangzhou metro museum," Archives Science Study, vol. 170, no. 5, pp. 100-103, 2019.

[2] D. B. Rawat, "Fusion of software defined networking, edge computing, and blockchain technology for wireless network virtualization," IEEE Communications Magazine, vol. 57, no. 10 , pp. 50-55, 2019.

[3] J. W. Wang, "Research on museum information and intelligent service mode based on internet of things technology," Information Science, vol. 38, no. 11, pp. 47-52, 2020.

[4] J. Y. Zhou, "Integration mode construction of LAM digital resource based on linked data," Library, vol. 292, no. 01, pp. 74-79, 2019.

[5] H. Chen, "Analysis on interaction design requirements of meishan nuo mask exhibits function entrance based on hunan museum APP," Packaging Engineering, vol. 40, no. 24, pp. 352-357, 2019.

[6] D. Bui-Thi, P. Meysman, and K. Lauke Ns, "Clustering association rules to build beliefs and discover unexpected patterns," Applied Intelligence, vol. 50, no. 2, pp. 1943-1954, 2020.

[7] P. Qin, M. Xiang, X. Liang, and Z. Hou, "A new method to integrate different gravity gradient components in reweighted regularized inversion with a minimum support constraint," Geophysical Journal International, vol. 217, no. 2, pp. 13871412, 2019.

[8] R. Bao, L. Chen, and P. Cui, "User behavior and user experience analysis for social network services," Wireless Networks, vol. 1 , no. 1, pp. 1-7, 2020.

[9] G. Xu, L. Zhang, C. Ma, and Y. Liu, "A mixed attributes oriented dynamic SOM fuzzy cluster algorithm for mobile user classification," Information Sciences, vol. 515, no. 3, pp. 280-293, 2019.

[10] C. H. Lai and C. Y. Hsu, "Rating prediction based on combination of review mining and user preference analysis," Information Systems, vol. 99, Article ID 101742, 2021.

[11] V. R. Fernando, "Modified integral action with time-varying forgetting function for tracking control," Proceedings of the Institution of Mechanical Engineers, Part I: Journal of Systems and Control Engineering, vol. 234, no. 6, pp. 715-725, 2019.

[12] S. Dhelim, N. Aung, and H. Ning, "Mining user interest based on personality-aware hybrid filtering in social networks," Knowledge-Based Systems, vol. 206, no. 3, Article ID 106227, 2020.

[13] H. Li, H. Duan, Y. Zheng, Q. Wang, and Y. Wang, “A CTR prediction model based on user interest via attention mechanism," Applied Intelligence, vol. 50, no. 4, pp. 1192-1203, 2020.

[14] X. Chao, Y. Dong, G. Kou, and Y. Peng, "How to determine the consensus threshold in group decision making: a method based on efficiency benchmark using benefit and cost insight," Annals of Operations Research, vol. 26, no. 12, pp. 23-30, 2021.

[15] M. J. Rezaee, M. Eshkevari, M. Saberi, and O. Hussain, "GBKmeans clustering algorithm: an improvement to the K-means algorithm based on the bargaining game," Knowledge-Based Systems, vol. 213, no. 6, Article ID 106672, 2021.
[16] D. Walsh, M. M. Hall, P. Clough, and J. Foster, "Characterising online museum users: a study of the national museums liverpool museum website," International Journal on Digital Libraries, vol. 21, no. 1, pp. 75-87, 2020.

[17] N. Grincheva, "The online museum: a "placeless" space of the "civic laboratory"” Museum Anthropology Review, vol. 8, no. 1, pp. 1-21, 2014. 